\title{
Best upper and lower bounds on Spearman's rho for zero-inflated continuous variables and their application to insurance
}

\author{
Mhamed Mesfioui \\ Département de mathématiques et d'informatique \\ Université du Québec à Trois-Rivières \\ Trois-Rivières (Québec) \\ Canada G9A 5H7 \\ Julien Trufin \\ Department of Mathematics \\ Université Libre de Bruxelles (ULB) \\ Bruxelles, Belgium
}

September 14, 2021 


\begin{abstract}
In this note, we establish the best lower and upper bounds on Spearman's rho for zeroinflated continuous random variables studied in Pimentel (2009). The proposed bounds are explicitly expressed in terms of the respective probability masses at the origin. As illustrated in an example based on insurance data, these bounds are useful in practice when interpreting the values of Spearman's rho.
\end{abstract}

Keywords: Spearman's rho, bivariate zero-inflated data, Frechet-Hoeffding bounds. 


\section{Introduction}

Zero-inflated (or zero-adjusted) distributions are often encountered in insurance. They combine a continuous distribution on the positive real line and a point mass at zero. In insurance studies, the total claim amount related to a given contract is often equal to 0, when no claims have been filed against the insurer, but may also be strictly positive when one or several accidents occurred. The total claim cost is thus decomposed into the product of an indicator for the event "the policy produces at least one claim during the reference period" and a positive random variable representing the total claim amount produced by the policy when at least one claim has been filed.

There are many applications in insurance where we aim to measure the dependency between zero-inflated distributions. We can mention the need to assess the dependency between annual claim amounts related to different guarantees or products owned by the same policyholder or between annual claim amounts related to policyholders belonging to the same household as discussed for instance in Pechon et al. (2020).

Kendall's tau and Spearman's rho are classical measures of association between distributions. However, when at least one of the distributions is non-continuous, such as in the case of zero-inflated distributions, we know from Nešlehová (2007) that Kendall's tau and Spearman's rho are restricted to sub-intervals of $[-1,1]$. Therefore, small positive values of Kendall's tau or Spearman's rho may actually support a strong positive dependence when getting close to its highest attainable value.

Denuit and Mesfioui (2017) derived the best lower and upper bounds on Kendall's tau for bivariate zero-inflated distributions proposed by Pimentel et al. (2015). In this note, we follow the same approach as in Denuit and Mesfioui (2017) to derive the best lower and upper bounds on Spearman's rho for bivariate zero-inflated distributions proposed by Pimentel (2009). We illustrate the use of these bounds when assessing the dependency between the annual claim costs of two guarantees owned by the same policyholder in car insurance. In this setting, we show that the upper bound derived in this note may change the conclusions of the analyst when interpreting the values of Spearman's rho.

This note is organized as follows. Sections 2 and 3 are respectively devoted to the notion of zero-inflated distributions and Spearman's rho for bivariate zero-inflated distributions. In Section 4, we derive the best lower and upper bounds on Spearman's rho for bivariate zeroinflated distributions. The final section deals with a numerical illustration performed on real data coming from a motor insurance portfolio of European policies with two guarantees.

\section{Zero-inflated distributions}

In this note, we consider non-negative random variables $X$ having a continuous distribution on $\mathbb{R}^{+}$with probability density function $h_{1}$ and a positive probability mass at $0, \mathrm{P}[X=$ $0]=p_{1}>0$. As indicated in Pimentel et al. (2015) and in Denuit and Mesfioui (2017), the distribution function of $X$ is then given by

$$
F(s)=p_{1} \mathrm{I}[s=0]+\left\{p_{1}+\left(1-p_{1}\right) F_{h_{1}}(s)\right\} \mathrm{I}[s>0],
$$

where I[.] stands for the indicator function and $F_{h_{1}}$ denotes the distribution of the random variable $X \mid X>0$. Recall that the generalized inverse of $F$ is defined by $F^{-1}(\alpha)=$ 
$\inf \{x \geq 0 \mid F(x) \geq \alpha\}, \alpha \in[0,1]$, and can be expressed as

$$
F^{-1}(\alpha)=F_{h_{1}}^{-1}\left(\frac{\alpha-p_{1}}{1-p_{1}}\right) \mathrm{I}\left[p_{1}<\alpha \leq 1\right],
$$

where $F_{h_{1}}^{-1}$ denotes the inverse of the function $F_{h_{1}}$.

\section{Spearman's rho for bivariate zero-inflated data}

Let $(X, Y)$ be a pair of random variables with joint distribution function $H$, i.e. $H(x, y)=$ $\mathrm{P}[X \leq x, Y \leq y]$. Suppose further that $X$ and $Y$ are zero-inflated continuous and nonnegative with respective distribution functions $F$ and $G$, and positive probability masses at 0 . Specifically, the distribution function $F$ of $X$ is given in (2.1) and $G$ is defined similarly by

$$
G(s)=p_{2} \mathrm{I}[s=0]+\left\{p_{2}+\left(1-p_{2}\right) G_{h_{2}}(s)\right\} \mathrm{I}[s>0]
$$

where $G_{h_{2}}$ is the distribution function of $Y$ given $Y>0$.

Consider independent copies $\left(X_{1}, Y_{1}\right),\left(X_{2}, Y_{2}\right)$ and $\left(X_{3}, Y_{3}\right)$ of $(X, Y)$. The population version of Spearman's rho is defined in terms of the probability of concordance minus the probability of discordance for the random pairs $\left(X_{1}, Y_{1}\right)$ and $\left(X_{2}, Y_{3}\right)$, that is,

$$
\rho_{H}=3\left(\mathrm{P}\left[\left(X_{1}-X_{2}\right)\left(Y_{1}-Y_{3}\right)>0\right]-\mathrm{P}\left[\left(X_{1}-X_{2}\right)\left(Y_{1}-Y_{3}\right)<0\right]\right) .
$$

An alternative formula for $\rho_{H}$ in terms of $H$ can be found in Mesfioui and Tajar (2005), that is,

$$
\rho_{H}=3\left(\mathrm{E}_{\Pi}\left[H\left(X^{-}, Y\right)\right]+\mathrm{E}_{\Pi}\left[H\left(X, Y^{-}\right)\right]+\mathrm{E}_{\Pi}\left[H\left(X, Y^{-}\right)\right]+\mathrm{E}_{\Pi}\left[H\left(X^{-}, Y^{-}\right)\right]-1\right),
$$

where $\Pi$ denotes the product probability distribution.

In the context of zero-inflated continuous and non-negative random pairs $(X, Y), \mathrm{Pi}$ mentel (2009) obtained an interesting and useful formula of the population version of Spearman's rho that is recalled next. Let us denote as $X_{10}$ a random variable distributed as $X$ given that $Y=0$, as $X_{11}$ a random variable distributed as $X$ given that $Y>0$, as $Y_{01}$ a random variable distributed as $Y$ given that $X=0$, as $Y_{11}$ a random variable distributed as $Y$ given that $X>0$, as $X_{1}$ a random variable distributed as $X$ given $X>0$, and as $Y_{1}$ a random variable distributed as $Y$ given that $Y>0$. Now, define $p_{00}=\mathrm{P}[X=0, Y=0]$, $p_{10}=\mathrm{P}[X>0, Y=0], p_{01}=\mathrm{P}[X=0, Y>0], p_{11}=\mathrm{P}[X>0, Y>0]$, and denote as $\rho_{11}$ Spearman's rho of $\left(X_{1}, Y_{1}\right)$. Then, $\rho_{H}$ can be expressed as

$$
\rho_{H}=\left(1-p_{1}\right)\left(1-p_{2}\right) p_{11} \rho_{11}+3\left(p_{00} p_{11}-p_{01} p_{10}\right)
$$

An estimator of $\rho_{H}$ based on (3.2) and its asymptotic distribution have also been proposed in Pimentel (2009). 


\section{General bounds on Spearman's rho}

Nešlehová (2007) established the following lower and upper bounds for Spearman's rho:

$$
\pm 3 \sqrt{\operatorname{var}(F(X-)+F(X)-1) \operatorname{var}(G(Y-)+G(Y)-1)} .
$$

A multivariate extension of these bounds has been established in Mesfioui and Quessy (2010). Note that the bounds (4.1) are useful in the situations where it is complicated to compute the best bounds for Spearman's rho based on Fréchet-Hoeffding bounds. In the context of zero-inflated continuous and non-negative random pairs $(X, Y)$, the bounds (4.1) reduce to

$$
\pm \sqrt{\left(1-p_{1}^{3}\right)\left(1-p_{2}^{3}\right)}
$$

which shows that the Spearman's rho (3.2) cannot reach the values \pm 1 when $p_{1}>0$ or $p_{2}>0$.

Proposition 2.6 in Mesfioui and Tajar (2005) enables to improve the bounds (4.2). It says that if $H_{1}$ and $H_{2}$ are two bivariate distribution functions such that $H_{1}(x, y) \leq H_{2}(x, y)$

for all $x$ and $y$, then $\rho_{H_{1}} \leq \rho_{H_{2}}$. Hence, the sharp lower and upper bounds of Spearman's rho can be derived using Fréchet-Hoeffding bounds, so that the bounds $\rho_{\min }$ and $\rho_{\max }$ on (3.2) can be computed by using the fact that $\rho_{\min }=\rho_{W}$ and $\rho_{\max }=\rho_{M}$ where $W$ and $M$ denote the Fréchet-Hoeffding bounds defined by $W(x, y)=\max \{F(x)+G(y)-1,0\}$ and $M(x, y)=\min \{F(x), G(y)\}$. Note that these sharp lower and upper bounds of Spearman's rho also appear in Nešlehová (2007). The next result establishes the expression of these bounds in terms of the probabilities $p_{1}$ and $p_{2}$.

Proposition 4.1. The lower and upper bounds on Spearman's rho are given by

$$
\begin{gathered}
\rho_{\max }=\left(1-\max \left(p_{1}, p_{2}\right)\right)\left[3 \min \left(p_{1}, p_{2}\right)+\left(1-p_{1}\right)\left(1-p_{2}\right)\right], \\
\rho_{\min }=\left(1-p_{1}-p_{2}\right)_{+}\left[3-\left(1-p_{1}\right)\left(1-p_{2}\right)\right]-3\left(1-p_{1}\right)\left(1-p_{2}\right),
\end{gathered}
$$

where $x_{+}=\max \{x, 0\}$.

Proof. As mentioned earlier the upper bound $\rho_{\max }$ on (3.2) is reached when the random pair $(X, Y)$ obeys the upper Frechet-Hoeffding bound, that is, when $X=F^{-1}(U)$ and $Y=G^{-1}(U)$, where $U$ is a random variable uniformly distributed over the unit interval $[0,1]$. Clearly, (2.2) shows that

$$
X=F^{-1}(U)=F_{h_{1}}^{-1}\left(\frac{U-p_{1}}{1-p_{1}}\right) \mathrm{I}\left[p_{1}<U \leq 1\right] \text { and } Y=G^{-1}(U)=G_{h_{2}}^{-1}\left(\frac{U-p_{2}}{1-p_{2}}\right) \mathrm{I}\left[p_{2}<U \leq 1\right] .
$$

Moreover, one observes that $\rho_{11}=1$ and

$$
\begin{aligned}
& p_{00}=\mathrm{P}[X=0, Y=0]=\mathrm{P}\left[U \leq \min \left(p_{1}, p_{2}\right)\right]=\min \left(p_{1}, p_{2}\right) \\
& p_{10}=\mathrm{P}[X>0, Y=0]=\mathrm{P}\left[p_{1}<U \leq p_{2}\right]=\left(p_{2}-p_{1}\right) \mathrm{I}\left[p_{2}>p_{1}\right] \\
& p_{01}=\mathrm{P}[X=0, Y>0]=\mathrm{P}\left[p_{2}<U \leq p_{1}\right]=\left(p_{1}-p_{2}\right) \mathrm{I}\left[p_{1}>p_{2}\right] \\
& p_{11}=\mathrm{P}[X>0, Y>0]=\mathrm{P}\left[U>\max \left(p_{1}, p_{2}\right)\right]=1-\max \left(p_{1}, p_{2}\right) .
\end{aligned}
$$


These imply that

$$
\rho_{\max }=\left(1-p_{1}\right)\left(1-p_{2}\right)\left(1-\max \left(p_{1}, p_{2}\right)\right)+3 \min \left(p_{1}, p_{2}\right)\left(1-\max \left(p_{1}, p_{2}\right)\right)
$$

which reduces to (4.3).

To establish the expression for the lower bound $\rho_{\min }$, we first calculate the probabilities $p_{00}, p_{10}, p_{01}$ and $p_{11}$ when $X$ and $Y$ obey the lower Frechet-Hoeffding bound, that is, $X=$ $F^{-1}(U)$ and $Y=G^{-1}(1-U)$. When $1-p_{1}-p_{2}<0 \Leftrightarrow p_{1}+p_{2}>1$, we get

$$
\begin{aligned}
& p_{00}=\mathrm{P}[X=0, Y=0]=\mathrm{P}\left[U \leq p_{1}, 1-U \leq p_{2}\right]=\mathrm{P}\left[1-p_{2} \leq U \leq p_{1}\right]=p_{1}+p_{2}-1 \\
& p_{10}=\mathrm{P}[X>0, Y=0]=\mathrm{P}\left[U>p_{1}, 1-U \leq p_{2}\right]=\mathrm{P}\left[U>p_{1}\right]=1-p_{1} \\
& p_{01}=\mathrm{P}[X=0, Y>0]=\mathrm{P}\left[U \leq p_{1}, 1-U>p_{2}\right]=\mathrm{P}\left[U \leq 1-p_{2}\right]=1-p_{2} \\
& p_{11}=\mathrm{P}[X>0, Y>0]=\mathrm{P}\left[U>p_{1}, 1-U>p_{2}\right]=0 .
\end{aligned}
$$

Using the fact that $\rho_{11}=-1$, it follows from (3.2) that

$$
\rho_{\min }=-3 p_{01} p_{10}=-3\left(1-p_{1}\right)\left(1-p_{2}\right) .
$$

Now, when $1-p_{1}-p_{2} \geq 0$, it comes $p_{00}=0, p_{10}=p_{2}, p_{01}=p_{1}$ and $p_{11}=1-p_{1}-p_{2}$. Hence, since $\rho_{11}=-1$ when $X$ and $Y$ are perfectly negatively dependent, (3.2) leads to

$$
\begin{aligned}
\rho_{\min } & =-\left(1-p_{1}\right)\left(1-p_{2}\right)\left(1-p_{1}-p_{2}\right)-3 p_{1} p_{2} \\
& =-\left(1-p_{1}\right)\left(1-p_{2}\right)\left(1-p_{1}-p_{2}\right)+3\left(1-p_{1}-p_{2}\right)-3\left(1-p_{1}\right)\left(1-p_{2}\right) \\
& =\left(1-p_{1}-p_{2}\right)\left[3-\left(1-p_{1}\right)\left(1-p_{2}\right)\right]-3\left(1-p_{1}\right)\left(1-p_{2}\right),
\end{aligned}
$$

which ends the proof.

Notice that the sharp upper bound (4.3) coincides with the upper bound (4.2) when $p_{1}=p_{2}=p$. Indeed, in such a case, we directly get

$$
\rho_{\max }=(1-p)\left\{3 p+(1-p)^{2}\right\}=(1-p)\left(1+p+p^{2}\right)=1-p^{3} .
$$

Otherwise, when $p_{1} \neq p_{2}$, these bounds can be quite different as illustrated in Figure 4.1 where $\rho_{\max }$ and $\sqrt{\left(1-p_{1}^{3}\right)\left(1-p_{2}^{3}\right)}$ are displayed in terms of $\left.p \in\right] 0,1\left[\right.$ for $p_{1}=p$ and $p_{2}=$ $1-p$. One sees that the sharp upper bound can significantly improve the upper bound (4.2) proposed in the literature.

Similarly, the sharp lower bound $\rho_{\min }$ can considerably improve the lower bound $-\sqrt{\left(1-p_{1}^{3}\right)\left(1-p_{2}^{3}\right)}$ as illustrated in Figure 4.2 for $p_{1}=p_{2}=p$. 


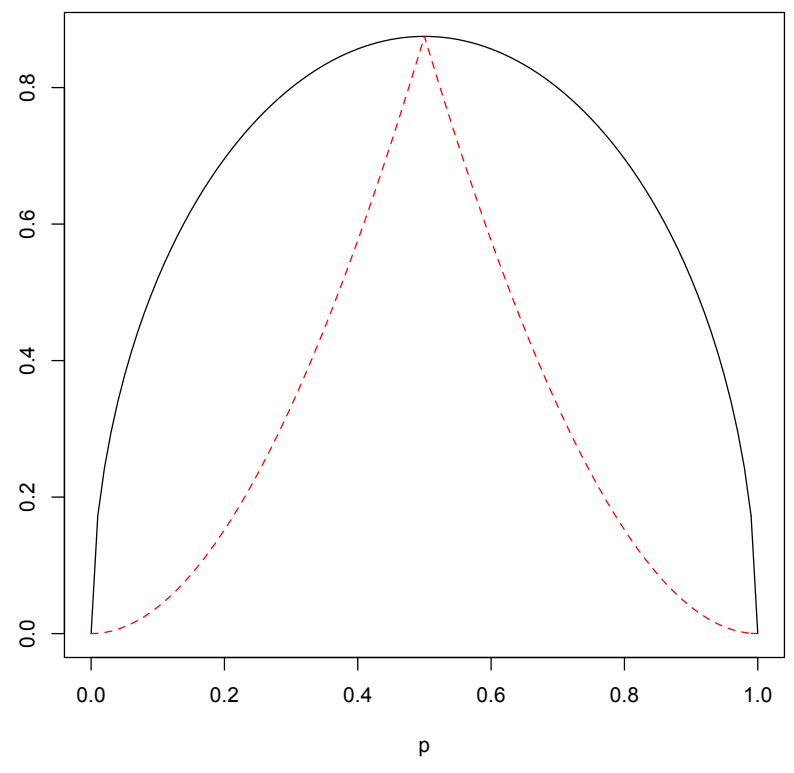

Figure 4.1: Bounds $\rho_{\max }$ (dashed line) and $\sqrt{\left(1-p_{1}^{3}\right)\left(1-p_{2}^{3}\right)}$ (solid line) in terms of $\left.p \in\right] 0,1[$ with $p_{1}=p$ and $p_{2}=1-p$.

\section{Numerical illustration}

The data relate to a motor insurance portfolio of an insurance company operating in the EU with two guarantees. This portfolio has been observed in 2019 and comprises 1312 policies (all observed during the whole year). The guarantees under consideration are the Third-Party Liability (TPL) insurance and the Material Damage (MD) insurance. TPL insurance is compulsory and covers a third-party's loss caused by the insured car. MD is an optional guarantee that covers the cost of repairing or replacing the insured's own vehicle. Typically, this guarantee is triggered when the policyholder is liable for the claim or could not identify the liable person. For each policy, we have at our disposal the annual claim costs by guarantee.

The estimates of the probability masses at 0 for both guarantees are $\hat{p}_{1}=0.965701$ (TPL) and $\hat{p}_{2}=0.873476(\mathrm{MD})$, and Figure 5.1 depicts the estimates for the distribution functions $F_{h_{1}}(\mathrm{MTPL})$ and $G_{h_{2}}(\mathrm{MD})$. There are thus many zero values for both guarantees, which is typical in insurance. The estimated Spearman's rho $\hat{\rho}_{H}$ is equal to 0.055626 and its corresponding estimated upper bound $\hat{\rho}_{\max }$ is equal to 0.090026. Therefore, $\hat{\rho}_{H}$ should not be compared to 1 but only to about 0.1 , so that one sees that both guarantees are actually significantly positively dependent.

\section{Acknowledgements}

Mhamed Mesfioui acknowledges the financial support of the Natural Sciences and Engineering Research Council of Canada No 261968-2013. 


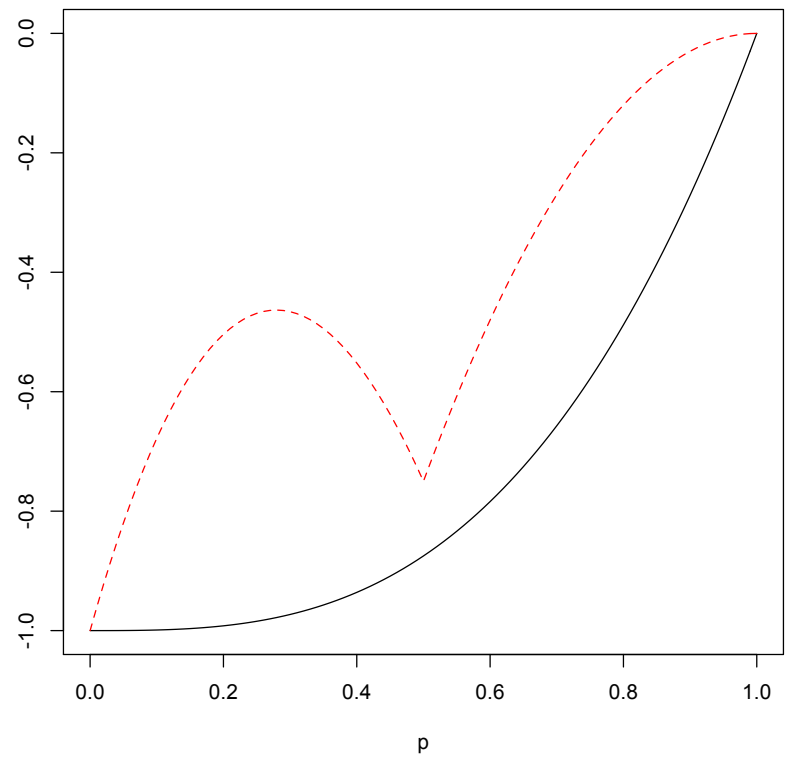

Figure 4.2: Bounds $\rho_{\min }$ (dashed line) and $-\sqrt{\left(1-p_{1}^{3}\right)\left(1-p_{2}^{3}\right)}$ (solid line) in terms of $p \in$ ]0, $1\left[\right.$ when $p_{1}=p_{2}=p$.

\section{References}

Denuit, M., Mesfioui, M. (2017). Bounds on Kendall's tau for zero-inflated continuous variables. Statistics and Probability Letters, 126, 173-178.

Mesfioui, M., Quessy, J. F. (2010). Concordance measures for multivariate non-continuous random vectors. Journal of Multivariate Analysis 101, 2398-2410.

Mesfioui, M., Tajar, A. (2005). On the properties of some nonparametric concordance measures in the discrete case. Nonparametric Statistics 17, 541-554.

Nešlehová, J. (2007). On rank correlation measures for non-continuous random variables. Journal of Multivariate Analysis, 98(3), 544-567.

Pechon, F., Denuit, M., Trufin, J. (2021). Home and Motor insurance joined at a household level using multivariate credibility. Annals of Actuarial Science 15(1), 82-114.

Pimentel, R.S. (2009). Kendall's Tau and Spearman's Rho for Zero Inflated Data (Ph.D. dissertation). Western Michigan University, Kalamazoo, Michigan.

Pimentel, R. S., Niewiadomska-Bugaj, M., Wang, J. C. (2015). Association of zero-inflated continuous variables. Statistics and Probability Letters 96, 61-67. 


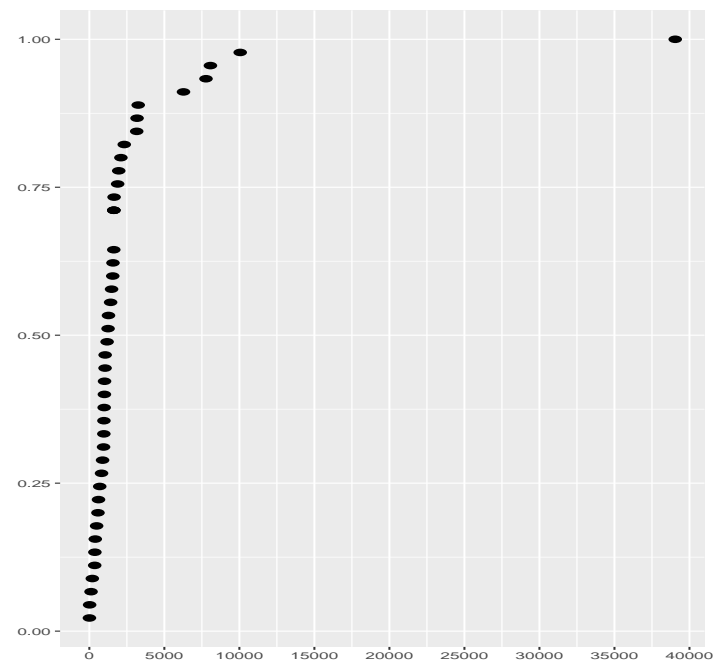

(a) TPL insurance: $\hat{F}_{h_{1}}$.

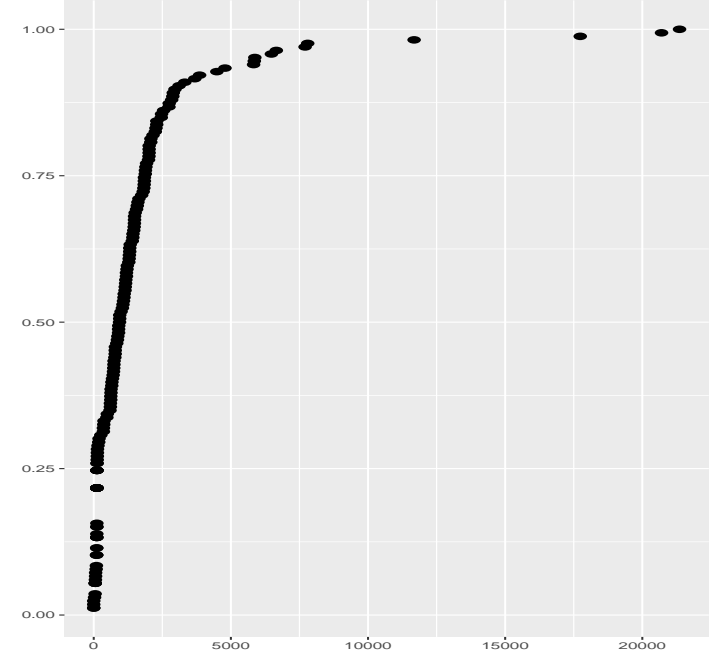

(b) MD insurance: $\hat{G}_{h_{2}}$.

Figure 5.1: Estimates of the distribution functions $F_{h_{1}}$ and $G_{h_{2}}$. 